\title{
The effect of absolute size on the separability of the dimensions of size and brightness
}

\author{
W. R. GARNER \\ Yale University, New Haven, Connecticut 06520
}

\begin{abstract}
Stimulus dimensions of size and brightness were used in an experimental paradigm which determines whether redundant dimensions are discriminated more easily than single dimensions and whether selective attention to dimensions is possible. Four different absolute sizes were used. The results show that selective attention is possible and that there is no facilitation of discrimination with redundant dimensions, and that these results are uninfluenced by absolute size. Thus, size and brightness are separable dimensions for all absolute sizes tested.
\end{abstract}

Garner and Felfoldy (1970) first used an experimental paradigm designed to distinguish between different types of dimensional interaction in information processing tasks. Briefly, a stimulus set of four stimuli generated from the orthogonal combinations of two levels on each of two dimensions is used. Tasks requiring discrimination of two stimuli are used as well as tasks requiring classification of all four stimuli. Discrimination tasks may involve differences on only a single dimension or on two correlated or redundant dimensions. If discrimination is faster with correlated dimensions than with single dimensions, then there has been a gain due to redundancy. The classification task requires that one dimension be relevant and the other irrelevant. If this task is performed as fast as the unidimensional tasks, then there has been selective attention to the relevant dimension. If the classification task is performed more slowly, then there has been a failure of selective attention.

Garner $(1974,1976)$ has summarized results from many experiments using this paradigm, and several types of dimensional interaction have been identified. Two of them are of primary importance in this paper: (1) Integral dimensions show improved discrimination with redundant dimensions and a failure of selective attention. (2) Separable dimensions show no improvement in discrimination of redundant dimensions, but allow selective attention to dimensions. Various conjectures have been made about what properties of the stimuli or their dimensions make for dimensional integrality or separability, and these are discussed by Garner (1974, p 136-138). Of special importance in this paper is the suggestion by Lockhead (1966) that integral dimensions must coexist in the same place and time.

The dimensions of size and brightness have never

This research was supported by Grant MH 14229 from the National Institute of Mental Health to Yale University. Maggie Davis served as the experimenter in all experiments and assisted in data analysis. been investigated with the full experimental paradigm, although research relevant to their integrality or separability has been done. Gottwald and Garner (1975) used the classification task and found no interference for either size or brightness. Thus, these dimensions would appear to be separable. Furthermore, Handel and Imai (1972) used these dimensions in a similarity judgment experiment, and found that a city-block metric rather than a euclidean metric best described their results. Since there is general evidence that separable dimensions do result in the city-block metric (again see Garner, 1974, Lecture 5, for summary), this result again suggests that size and brightness are separable. However, Biederman and Checkosky (1970) found a redundancy gain with these dimensions in a discrete reaction time task. The redundancy gain was very small (under $20 \mathrm{msec}$ ), so the evidence for integrality is not strong. However, even though visual angles of stimuli were not exactly specified, it seems clear that these authors had used smaller stimuli than were used by either Gottwald and Garner (1975) or by Handel and Imai (1972). If coexistence of dimensions in time and space is an important factor in producing the integral relation, then possibly absolute size of stimuli would have some effect on whether dimensions are integral or separable.

The specific purpose of the present experiment is to test the idea that absolute size is a determiner of dimensional integrality. The dimensions used were size and brightness, but with four subexperiments involving four different absolute sizes of stimulus. The specific expectation was that larger absolute sizes would give a separable result, while smaller absolute sizes would give an integral result.

\section{METHOD}

\section{Stimulus Conditions}

All stimuli were gray circular patches mounted in the center of a $6.3 \times 8.9-\mathrm{cm}$ white card, with the upper right corner missing to maintain proper orientation. The card was covered with a thin plastic to avoid soiling and to allow easy sorting. The circles on the cards varied in two dimensions: brightness and 
size. Within each of the four subexperiments, there were four different stimuli formed by the orthogonal combinations of two levels on each of the two dimensions. These stimuli can be designated by combining the terms B1 and B2 for the lighter and darker brightnesses, respectively, with the terms S1 and S2 for the smaller and larger sizes. Thus, the four stimuli are designated B1S1, B1S2, B2S1, B2S2.

Brightness levels for each subexperiment were Munsell values 5 and 6. Since the stimuli were placed on white cards, the circular patches were darker than the background.

Relative sizes within each subexperiment were such that the larger stimulus had a diameter 1.1875 times that of the smalle stimulus.

Absolute size was different for each subexperiment and is most easily specified as the diameter of the smaller circle. These were $4.0,8.0,16.0$, and $32.0 \mathrm{~mm}$. Since these stimuli are typically viewed from a distance of about $30 \mathrm{~cm}$, the smallest size used subtends a visual angle of less than $1 \mathrm{deg}$ and the largest size subtends an angle greater then 7 deg.

\section{Tasks}

Each task required the subject to sort a deck of 32 stimulus cards into two piles as rapidly and as accurately as possible. There were eight different tasks of two basically different types:

Discrimination tasks required the subject to classify a deck of just two stimulus alternatives (16 of each in the deck). There were six pairs of four stimuli, and all were used. The tasks can be described by noting the stimuli involved, with a slash to indicate that the stimuli are to be classified differently. There were two tasks which required just brightness discrimination: B1S1/B2S1 and $B 1 S 2 / B 2 S 2$. There were two tasks which required just size discrimination: $\mathrm{B} 1 \mathrm{~S} 1 / \mathrm{B} 1 \mathrm{~S} 2$ and $\mathrm{B} 2 \mathrm{~S} 1 / \mathrm{B} 2 \mathrm{~S} 2$. There were two tasks which required discrimination of correlated or redundant dimensions: B1S1/B2S2 and B2S1/B1S2.

Classification tasks required the subject to sort all four stimuli (eight of each in each deck) into two piles. One task required classification by brightness, with size an irrelevant dimension: B1S1, B1S2/B2S1, B2S2. The other task required classification by size, with brightness an irrelevant dimension: B1S1, B2S1/B1S2, B2S2.

\section{Procedure}

On each trial the subject was handed a shuffled deck of cards, face up, along with the two or four example cards indicating the particular classification required on that trial. The experimenter said "ready," then "set," and then started a stopwatch. The click of the stopwatch was the signal for the -subject to begin sorting. After each sort the subject was told the sorting time, the subject himself reported any errors, and the next trial was begun. The experiment was carried out in an office, with the subject sitting across a table from the experimenter, who operated the stopwatch, shuffled decks, and handed them to the subject, did all the recording and gave feedback at the end of the session. Times were recorded to the nearest tenth of a second.

\section{Subjects and Design}

A total of 32 subjects were used, the majority of them satisfying an undergraduate course requirement, with a few graduate students who were paid, all at Yale University. Eight different subjects were used for each subexperiment.

Each subject was run on all eight tasks for one subexperiment. On block of trials involved all tasks. A practice block of trials was run to familiarize the subject with the tasks, and then four data blocks were run. The total session took about $1 \mathrm{~h}$. For each subexperiment two semirandom sequences of tasks were used (one for each of four subjects). The sequences were constrained so that the different types of tasks were not heavily represented in early or late stages. A sequence was used in the first block, then reversed in the second block, and again in the third and fourth blocks.

\section{Data Analyses}

As is typical in such experiments, errors were too few to analyze. Many different analyses of variance were carried out on the sorting times, and statements of effects will be based on a significance level of .01 unless otherwise specified. In addition, some nonparametric tests were used to check specific points.

\section{RESULTS}

\section{Effects of Absolute Size}

Mean sorting times (across subjects and blocks) are shown in Table 1 for all tasks and absolute sizes of stimuli. There is a general decrease in sorting time as absolute size is increased, but this effect is not statistically significant as it stands. However, the linear component of sorting time vs either size of smaller stimulus or the logarithm of that size is significant at the .05 level. Thus, the effect of absolute size within these ranges is only marginally significant.

Of more importance is the question of the interaction of Absolute Size by Tasks. This interaction is not significant $(F<1.0)$, nor is the interaction of the linear trend across sizes with tasks significant. Thus, while there is some slight evidence of improvement in classification speed with larger sizes, this improvement operates equally for both brightness and size discrimination, as well as for redundant discrimination and both types of classification. The actual slopes of these linear functions with respect to the logarithm of smaller size for the eight different tasks range only from -.30 to -.45 , and there is no systematic change in these slopes. To illustrate, the smallest slope and the next to steepest slope occur with the two tasks requiring simple brightness discrimination. These results indicate that we can

Table 1

Mean Sorting Time (seconds) for Eight Size and/or Brightness Tasks at Four Absolute Sizes of the Smaller Stimulus

Size of Smaller Stimulus (in millimeters)

\begin{tabular}{llllll}
\cline { 2 - 5 } Task & 4 & 8 & 16 & 32 & Mean \\
\hline
\end{tabular}

Brightness Discrimination

B1S1/B2S1

B1S2/B2S2

$\begin{array}{llll}14.30 & 14.38 & 13.78 & 13.52\end{array}$

$\begin{array}{lllll}14.49 & 14.75 & 13.80 & 13.34 & 14.10\end{array}$

Mean

$\begin{array}{lllll}14.40 & 14.57 & 13.79 & 13.43 & 14.05\end{array}$

Size Discrimination

B1S1/B1S2

B2S1/B2S2

$\begin{array}{llll}14.67 & 14.82 & 13.87 & 13.49\end{array}$

14.21

Mean

$\begin{array}{llllll}14.85 & 15.11 & 14.03 & 13.70 & 14.42\end{array}$

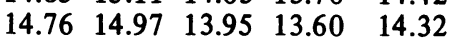

Redundant Discrimination

B1S1/B2S2

B2S1/B1S2

$\begin{array}{llll}14.20 & 14.52 & 13.43 & 13.30\end{array}$

13.86

Mean

$\begin{array}{llllll}14.33 & 14.39 & 13.45 & 13.40 & 13.89\end{array}$

$\begin{array}{lllll}14.26 & 14.45 & 13.44 & 13.35 & 13.88\end{array}$

Classification

Brightness

Size

Mean

$\begin{array}{llll}14.94 & 15.04 & 14.24 & 13.85\end{array}$

14.52

$\begin{array}{lllll}15.07 & 15.42 & 14.16 & 14.19 & 14.71\end{array}$

Overall Mean 
deal with the differences between tasks without regard to the absolute size of stimuli involved.

\section{Effect of Tasks}

There are significant differences between the eight tasks. These eight tasks, however, consist of four different types of task and two specific tasks within each type. The variance due to differences between specific tasks within type of task is very small and nonsignificant. We can thus ignore differences between specific tasks with each type of task.

Unidimensional discrimination. On the other hand, differences between types of task are substantial and are statistically significant. One of the significant differences is that, in simple discrimination, size differences give slower performance than brightness differences. The levels on each of the two dimensions were selected with the intent of providing equally difficult discrimination, but the failure to achieve it is slight, being only $.27 \mathrm{sec}$.

Redundant discrimination. If the two dimensions are integral, then the redundant discrimination tasks should be performed better than the unidimensional tasks. The average of the two redundant tasks is indeed faster than either the brightness or size discriminations. This redundancy gain is statistically significant when measured against the poorer of the two unidimensional discriminations (size), but is not statistically significant when measured against the better of the two unidimensional discriminations (brightness). Furthermore, it is preferable in determining whether a redundancy gain exists to compare the redundant conditions against the better dimension for each subject, since all subjects may not agree on which dimension is easier. When the average of the two redundant discriminations is compared to the better of the average of either the two brightness discriminations or the two size discriminations for each subject, then the redundancy gain disappears entirely, with a mean gain of $.04 \mathrm{sec}$ and with only 16 of the 32 subjects showing better performance with redundant dimensions. Thus, we may conclude that there is no advantage to the use of redundant dimensions of size and brightness.

Selective attention. The ability to attend selectively to the dimensions is measured by the relative loss of sorting time in the classification tasks compared to the unidimensional discriminations. Both brightness and size classification are slower than their unidimensional counterparts. Once again, however, the effect is slight, being less than $.5 \mathrm{sec}$ in both cases. This much interference is considerably less than is obtained with integral dimensions such as Munsell value and chroma, with which the loss of sorting speed is between 3 and $4 \mathrm{sec}$ (Garner \& Felfoldy, 1970). Thus, the interference found in the present experiment is very weak and is probably due to a general disruption effect caused by stimulus variability rather than to a true failure of selective attention.
In summary, these analyses indicate that the dimensions of size and brightness are separable, and that this relation is not influenced by the overall sizes of the stimuli.

\section{Effects of Learning}

The above analyses were performed on data averaged over four blocks of trials. In case there were effects lost in this overall analysis, an additional analysis was carried out with data from the first and fourth blocks of trials, to see if any of the effects described above had changed over the time course of the experiment. There was a significant decrease in average sorting time of almost $1.0 \mathrm{sec}$ between the two blocks. There was, however, no interaction of Blocks by Absolute Size, either of the simple effects of absolute size or of the linear component.

Of greater importance was any possible change in task effects over the course of the experiment. The interaction of Blocks by Tasks was not significant $(F<1.0)$. The fact that the change from the first to the fourth block is almost entirely due to a simple average decrease in sorting time can be demonstrated by noting that the means for the four task types decreased by $.88, .96, .99$, and $.98 \mathrm{sec}$ in order as the task types are shown in Table 1. Thus, the conclusions drawn from the analyses of average data are valid for any block of the data.

\section{CONCLUSIONS}

This experiment has shown more completely than has been shown before that the stimulus dimensions of brightness and size are separable. Furthermore, the separable relation between the dimensions is not a function of absolute size within the limits of these conditions. This experiment does not help clarify the conditions which produce integral stimulus dimensions.

\section{REFERENCES}

Biederman, I., \& Checkosky, S. F. Processing redundant information. Journal of Experimental Psychology, 1970, 83, 486-490.

GARNER, W. R. The processing of information and structure. Potomac, Maryland: Lawrence Erlbaum, 1974.

GARNER, W. R. Interaction of stimulus dimensions in concept and choice processes. Cognitive Psychology, 1976, 8, 98-123.

Garner, W. R., \& Felfoldy, G. L. Integrality of stimulus dimensions in various types of information processing. Cognitive Psychology, 1970, 1, 225-241.

GotTwald, R. L., \& Garner, W. R. Filtering and condensation tasks with integral and separable dimensions. Perception \& Psychophysics, 1975, 18, 26-28.

HANDEL, S., \& ImaI, S. The free classification of analyzable and unanalyzable stimuli. Perception \& Psychophysics, 1972, 12, 108-116.

LOCKHEAD, G. R. Effects of dimensional redundancy on visual discrimination. Journal of Experimental Psychology, 1966, 72, 95-104.

(Received for publication December 30, 1976.) 